\title{
An Artificial Intelligent and Big Data Research Platform Based on Jupyter Notebook
}

\author{
Ligu Zhu ${ }^{1}$, Suping Wang ${ }^{1+}$ \\ ${ }^{1}$ Communication University of China, Beijing Key Laboratory of Big Data in Security \& Protection Industry, \\ Supported by the Fundamental Research Funds for the Central Universities
}

\begin{abstract}
In the era of data-driven artificial intelligence technology, large depth of the neural network in the process of scientific research, the algorithm is to accelerate and improve step by step. The processing of such high performance computing and parallel computing ability are more important. This article introduces the big data intelligent science and technology system, mainly to solve the hardware sharing, users' requirements and the cloud application problems of individuation. Platform embeds Jupyter Notebook, provides data storage, data processing and data analysis function, and it can also provide deep learning training environment and improve the efficiency of training, so as to construct big data application's architecture of the intelligent system. Finally, the development and construction of micro-service architecture based on cloud computing infrastructure and the deployment delivery system based on Docker containerized application are realized.
\end{abstract}

Keywords: artificial intelligence, research platform, Jupyter notebook, hardware sharing, model training, Docker

\section{Introduction}

In recent years, the research on big data architecture and key technologies represented by containers, micro-service and componentization have attracted much attention. Big data intelligence mainly consists of three elements, namely "data", "algorithm" and "computing power". The explosive growth of data promotes the popularization and development of large-scale applications in the era of intelligence. The improvement and breakthrough of algorithms such as machine learning and deep learning expand the intelligent perception ability of application layer. GPU provides computing resources with high performance for the training process of deep learning algorithms.

The overall architecture of the artificial intelligence and big data research platform adopts the hierarchical form. It will be micro-service system at the same time, combining with the characteristics of Docker containers' advantage and extensive use of Docker containers' technology. The platform makes full use of computing resources in the meantime, aimed at simplifying the use of deep learning framework, realizing share of GPU core resources, and improving the utilization rate of resources and management of deep learning process of training model, management resources and distribution of cluster monitoring, so as to improve the effect of scientific research. ${ }^{1}$

The composition of the platform system is shown in figure 1 .

As can be seen from figure 1, the platform is mainly committed to solving three kinds of problems.

- How to organize the hardware reasonably.

\footnotetext{
+ Corresponding author. Tel.: +86-10-13021234104.

E-mail address:wspspring@126.com.
} 
Platform combines the CPU and GPU resources, so as to realize the reasonable utilization of the hardware computing resources, ultimately provide hardware to satisfy the needs for the researchers of each computing resources. Efficiently training process of deep learning algorithms and hardware sharing also solve the problem of the waste of resource when used alone.

- How to manage and schedule resources.

Platform provides two kinds of deep learning frameworks of prevailing standards, namely TensorFlow framework and PyTorch framework, at the same time combining Docker lightweight virtualization technology and Jupyter Notebook supports a variety of characteristics of kernel. In Jupyter Notebook, TensorFlow/PyTorch framework environment and dependencies on the middleware framework of resources are all packed into the mirror, for each of the scientific research personnel to run the image, so as to guarantee in the form of the Docker container model of scientific training process and algorithmic researches.

- How the platform interacts with users.

The platform provides service for researchers in the form of WEB UI service. Cloud applications are no longer limited to the software basic environment and are not restricted by geographical location. Whenever and wherever, as long as the users have a network, they can access to the platform. At the same time, after entering the TensorLFlow or PyTorch service, model training and research process of algorithms will be carried out through the real-time interactive WEB page of Jupyter Notebook. Meanwhile, Jupyter Notebook can meet users' personalized needs, such as different storage conditions, hardware and software conditions, and framework versions.

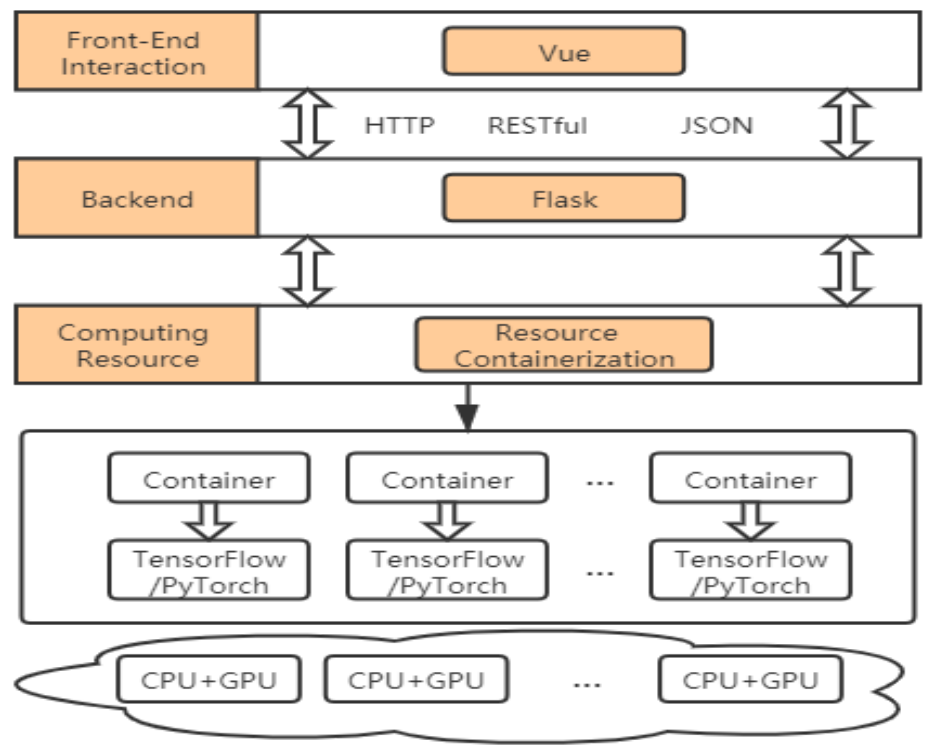

Fig. 1: The composition of the platform system

\section{Key Technology Stack}

\subsection{Jupyter Notebook}

Researchers use programming language which they are skilled in, so we need to provide a variety of programming environments, such as the Python programming language, R language and SQL language. Jupyter Notebook as an open source tool, provides more than 40 of the required kernel programming environments, provides the fusion of different data basic forms, and the interactive web pages for users, such as Numpy, Scikit - Learn, Matplotlib, and so on. It can be used for data analysis, visualization, and can also be effective in real-time coding, running and testing. It embeds narrative text, and the real-time visualization, the results of data preprocessing, simulation numerical, statistical and modelling, on machine learning and deep learning model for training and training results visualization. Therefore, the combination of Jupyter Notebook integrated environment and deep learning frameworks can provide a variety of deep learning research environments for different users. 
Jupyter Notebook technology stack is selected because its characteristics can meet the researchers' requirements for strong diversity and high availability of the environment:

- A variety of languages: no matter what kind of programming language researchers are good at, it can be installed the corresponding kernel for academic researches;

- Highly interactive function: researchers can install plug-ins to present some interactive visual content;

- The remote way of operation: researchers only need to through the WEB, can be through the network link remote server to achieve training and other scientific research, to avoid the problem of insufficient local computing resources.

- Sharing way is convenient: Notebook can not only support the form of web pages to share, can also be exported to a variety of formats of documents to share;

- Debugging is convenient: researchers only need to run all of the code once, it will save all the script content to memory, after the original code to add, delete, change operations, only need to run the update part, not all of them, you can get the result.

Based on Jupyter Notebook, deep learning, algorithm research, model training and application development can be completed. Application scenarios of Jupyter Notebook on the platform, including basic python programming courses, training of simple models, data analysis, etc. it can also be studied in the aspect of deep learning, model training, deep learning, image classification, object category detection, visualization homework, drawing graphs with a large amount of data. When researchers after upload training mission of deep learning, platform through the management node, as its load training data set, platform to realize data persisted, will all the data sets and model of scientific research personnel training script save, in the CPU and GPU computing resources of the infrastructure training missions, after the training model, and then the test link, namely the start loading test data sets, and then verifies the model, basic process following figure 2:

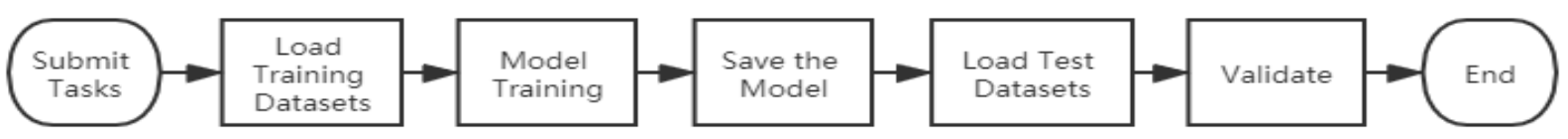

Fig. 2: Basic training process.

Jupyter Notebook adopts B/S (Browser/Server) architecture. After entering the interactive UI programming page of Jupyter Notebook, you can edit the code and submit the code to the kernel in real time to view the running results of the kernel in at once.

\subsection{Docker Container}

Docker container has the obvious characteristics of lightweight and isolation, which can simultaneously run tens of thousands of Docker containers on the same host machine, reasonably allocate the resources of the system, so as to achieve the highest operating efficiency of the system. It starts quickly, in a matter of seconds. The coupling between different Docker containers is extremely low, thus ensuring the independence of each module.

The research platform adopts the micro-service architecture of Docker technology, which can fully decouple the system. The development, deployment, upgrading, rollback, application self-recovery and other processes of the platform, and the source code is compiled and packaged with the help of Docker image, which is easy to manage the deployment.

The research platform will package all the dependencies of the Jupyter Notebook environment and the TensorFlow / PyTorch framework into the Docker image. When the researchers use the platform, they will immediately start the corresponding version of the TensorFlow / PyTorch container of the Jupyter Notebook, and load the persistent data files to meet the needs of researches.

\subsection{Vue and Flask}

The research platform adopts the separated technology of front-end and back-end for development. The front-end adopts the progressive JavaScript framework. Vue.js technology combines with the Element UI 
components. The cross-domain problem involved in the separation of front-end and back-end is solved by Axios. The technical features of Vue.js are the bi-directional binding, componentization and modularization of the data and view layers, which make the code highly reusable, highly readable and easy to manage. Flask is characterized by its lightweight and highly expandable technology.

\section{Construction and use of Jupyter Notebook}

The deep learning algorithms' framework which is provided by artificial intelligence and big data research platform mainly includes TensorFlow model framework and PyTorch model framework. Each framework environment is integrated in a specific mirror, which aims to provide complete resources and operating environment for researchers' model training tasks and other research work. There are three versions of the two deep learning training frameworks, including TensorFlow 1.13.0, TensorFlow 1.14.0, TensorFlow 2.0.0, PyTorch 1.1, PyTorch 1.2 and PyTorch 1.3. Researchers can choose their own version of the framework as needed.

\subsection{Construction of Jupyter Notebook}

Platform framework will be accompanied by Nvidia GPU driver Ubuntu16.04 system as a basic image, as a result of the Anaconda is Python integration platform, contains many Python common bags about data science, and can automatically install dependencies. As a result, platform in the process of making TensoFlow / PyTorch mirror, adds Anaconda3 environment, and configures the environment variables, on the basis of this installation TensorFlow / PyTorch, after installation requires Notebook configuration file modification, To make the environment work. After the successful installation, configuration and operation of all the environment and dependencies, the framework environment of TensorFlow / PyTorch deep learning algorithms that meet the requirements, is obtained. After the successful environment configuration, the generated Docker image is launched by the platform after the framework is selected by the researchers. Each user's Docker container runs independently without interference.

Because the generated Jupyter Notebook environment needs to be combined with the research platform, a number of configurations are needed in the process of integrating Jupyter Notebook in the platform.

- SSO single-sign-on authentication is used in the research platform, so in order to ensure the security of the platform and the permission authentication of the server on the Jupyter Notebook environment, the use of Notebook also needs to set the permission in the configuration file, which names jupyter_notebook_config.py.

- All the researchers' data, code and other files are persisted to the current user, so every time the user uses the TensorFlow or PyTorch framework, the platform will be the current user's files mounted to the current container. Every time the file is modified, added or deleted, it will be synchronized to the file storage system.

- Because the original Jupyter Notebook operating interface and research platform requirements are not consistent, therefore, the platform on the basis of the Notebook internal source code to make relevant adjustments, That is to python3.6/ site-packages /notebook/static directory under the tree/js/main.min.js, notebook/js/main.min.js, edit/js/main.min.js, auth/js/main.min.js, terminal/js/main.min.js, to ensure the normal use of platform Jupyter notebook.

- Because external system embedding is not allowed by default in Jupyter Notebook, it is necessary to set the CSP (Content Security Policy), that is, when starting Jupyter Notebook, set the parameters of the headers in parameters of c.notebookapp.tornado_settings, to set the research platform to allow its embedding, access and use.

- Every time each researcher opens the TensorFlow or PyTorch framework, the platform matches the corresponding Jupyter Notebook environment for each researcher, so that each researcher has its own deep learning framework environment of Jupyter Notebook, which is not affected by other personnel, nor will affect the model training and other research process of others. 


\subsection{Use of Jupyter Notebook}

In the research platform, the service module interface of the TensorFlow framework is shown in figure 3 below:

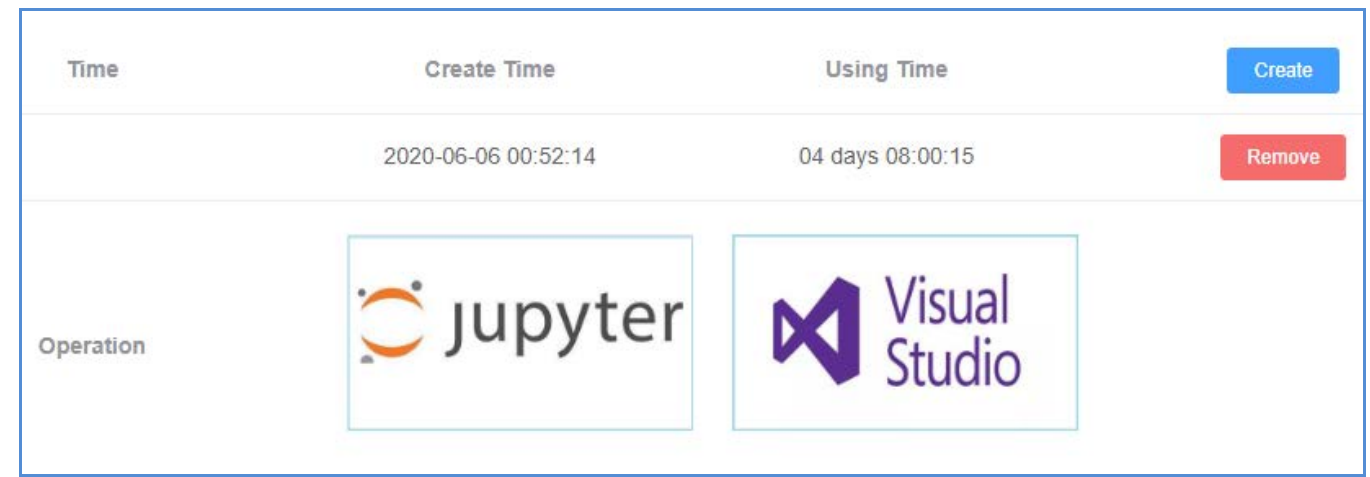

Fig. 3: The TensorFlow framework service module.

Click the "start creating" button on the page to jump to the TensorFlow framework creation page, as shown in figure 4 below. To create the TensorFlow framework, you need to fill in the basic information, including: name, description, model version, GPU specification.

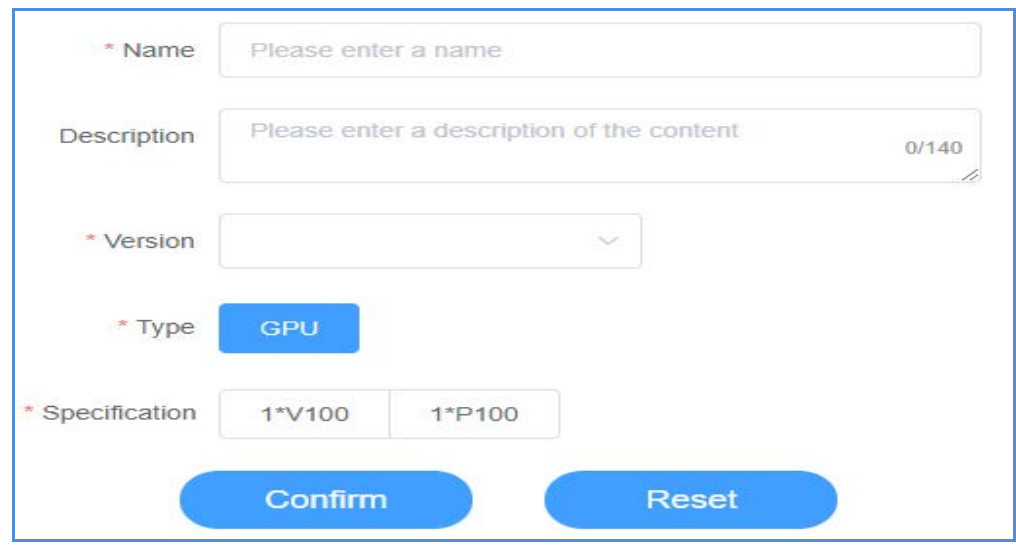

Fig. 4: The creation of TensorFlow framework.

After clicking the TensorFlow or PyTorch framework of the research platform, the Jupyter Notebook interface integrated with the TensorFlow or PyTorch framework can be opened and related operations can be conducted, as shown in figure 5 below:

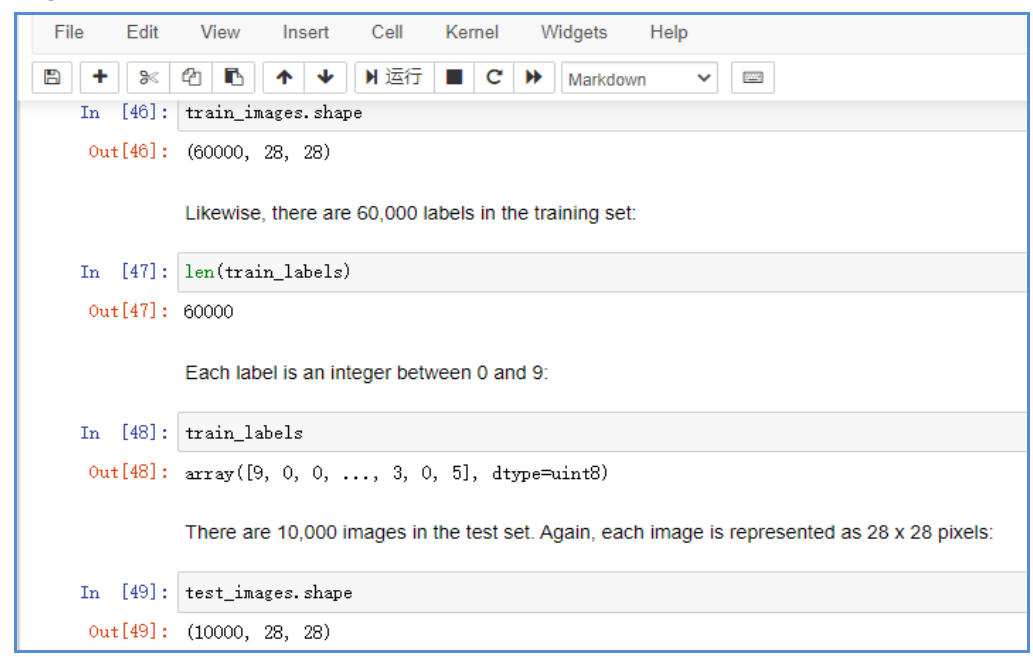

Fig. 5: the Encoding page. 


\section{Conclusion}

Using big data and artificial intelligence research platform, using micro-service way, combined with the deep learning TensorFlow framework, PyTorch framework to reconstruct Jupyter Notebook, research personnel can easily and quickly build machine learning and deep learning model, the model persistent deployment to the hosting environment, facilitate subsequent model optimization, data analysis , scientific research work and so on. In order to break through the massive data to deep learning algorithm performance bottlenecks in the process of training and hardware computing resources expensive problems, big data and artificial intelligence research platform provides an online model training environment, promote the scientific research personnel training process, thus lay a foundation for improving and upgrading the algorithm.

Of course, there are still shortcomings in the current research platform, such as the model training task can't be automated parameter adjustment, excellent adjustment. It's still necessary to continue to improve the platform.

\section{References}

[1] Lars Malmström. Computational Proteomics with Jupyter and Python[M]// Mass Spectrometry of Proteins. 2019.

[2] Boscoe, Bernadette M, Pasquetto, Irene V, Golshan, Milena S. Using the Jupyter Notebook as a Tool for Open Science: An Empirical Study[J].

[3] Amir Szitenberg, Max John, Mark L. Blaxter. ReproPhylo: An Environment for Reproducible Phylogenomics[J]. Plos Computational Biology, 2015, 11(9):e1004447.

[4] Xiaofeng Wang, Yewu Zhang, Yanfei Zhang. The Application of Jupyter Notebook in the Big Data Platform of Disease Control and Scientific Research[J]. Digital medicine in China, 2019, 014(005):118-120. 